\title{
Prevalência da síndrome cerebral orgânica em população de idosos de área metropolitana da região sudeste do Brasil
}

\author{
Prevalence of organic brain syndrome in an elderly population in a metropolitan \\ population of southeastern Brazil
}

Renato P. Veras*, Evandro Coutinho*

\begin{abstract}
VERAS, R.P. \& COUTINHO, E. Prevalência da síndrome cerebral orgânica em população de idosos de área metropolitana da região sudeste do Brasil. Rev. Saúde Pública, 28:26-37, 1994. Foram apresentados os principais resultados do estudo de prevalência de síndrome cerebral orgânica, realizado na cidade do Rio de Janeiro, com a população idosa. A partir de um conjunto de indicadores foram selecionados três distritos: Copacabana, Méier e Santa Cruz. No de Copacabana foi realizado o estudo de validação e confiabilidade do instrumento de diagnóstico. Os resultados de prevalência em Copacabana, Méier e Santa Cruz para sindrome cerebral orgânica foram de 5,9\%, 9,8\% e $29,7 \%$, respectivamente. Várias hipóteses foram formuladas para resultados tão díspares em uma mesma cidade. São apresentadas associações com vários indicadores socioeconômicos. Idosos com perda de autonomia e dependência são fortemente associados à sindrome cerebral orgânica.
\end{abstract}

Descritores: Distúrbios mentais orgânicos, epidemiologia. Depressão, epidemiologia. Idoso. Questionários.

\section{Introdução}

O crescimento demográfico brasileiro do grupo etário acima dos 60 anos $4,22,23,35,38,39,45,46,48$ levou alguns pesquisadores a buscar maiores informações sobre suas conseqüências no campo da saúde. Alguns projetos de maior monta foram desenvolvidos nas principais cidades brasileiras ${ }^{13,34,47}$. Nesses trabalhos, os problemas de saúde mental mereceram atenção especial.

O objetivo do presente artigo é apresentar os resultados de síndrome cerebral orgânica $(\mathrm{SCO})$ do segmento de saúde mental do estudo realizado na cidade do Rio de Janeiro, as correlações com os indicadores socioeconômicos e atividades do dia-adia do idoso, além das reflexões e recomendações.

Os aspectos metodológicos, o instrumento utilizado, a amostragem, o estudo piloto e o estudo de validação e confiabilidade já foram apresentados em outros artigos ${ }^{40,41,42,43,44}$ e não fazem parte do presente trabalho.

Instituto de Medicina Social da Universidade Estadual do Rio de Janeiro - Rio de Janeiro, RJ - Brasil

Separatas/Reprints: R.P.Veras - Rua São Francisco Xavier, 524 - $7^{2}$ andar - 20559-900 - Rio de Janeiro, RJ - Brasil.
Definição da área de estudo

Ao se escolher o Município do Rio de Janeiro, a intenção não foi trabalhar a cidade como um todo, o que levaria a conclusões equivocadas, dada a grande heterogeneidade de suas regiões. Por essa razão, optou-se por utilizar distritos que apresentassem certo grau de homogeneidade de sua população, do ponto de vista socioeconômico, e assim captar de forma mais adequada as necessidades reais e as demandas de seus diferentes grupos sociais. Entre todos os distritos do Municĺpio do Rio de Janeiro, Copacabana, Méier e Santa Cruz foram os que se enquadraram, a partir dos critérios definidos, como os representantes,

Tabela 1. Distribuição da situação socioeconômica dos distritos estudados.

\begin{tabular}{cccc}
\hline Categorias & Copacabana & Méier & Santa Cruz \\
socioeconómicas & $N=252$ & $N=244$ & $N=242$ \\
\hline Baixa (\%) & 2,0 & 10,2 & 36,0 \\
Média (\%) & 44,0 & 73,4 & 57,0 \\
Alta (\%) & 54,0 & 16,4 & 7,0 \\
\hline
\end{tabular}

Nota - Procedimento estatistico usado: Chi-Square, DF $=4$, Valor of $x^{2}=232,791, p<0,001$. 
respectivamente, das populações rica, média e pobre da cidade. Durante 14 meses de trabalho de campo , foram realizadas 252 entrevistas em Copacabana, 244 no Méier e 242 em Santa Cruz. O coeficiente de respostas foi de 93,3\%, 93,8\% e $93,1 \%$, respectivamente. Este alto índice de questionários completados deveu-se, entre outros motivos, ao trabalho extremamente competente da equipe e do supervisor de campo**. Pelo fato de se ter utilizado um questionário multidimensional ${ }^{8,40}$, o estudo gerou conjunto de dados, e os vários indicadores socioeconômicos, quando agrupados e confrontados com os distritos selecionados para o estudo, confirmaram a correção das áreas eleitas para o projeto. A Tabela 1 mostra a heterogeneidade entre os distritos estudados, confirmando a assunção de que os três distritos são distintos em termos socioeconômicos ${ }^{* * *}$.

\section{Apresentação do questionário de Saúde Mental - BOAS}

No segmento de saúde mental, a pesquisa dividiu-se em três fases: o piloto, o estudo de confiabilidade e validação do questionário BOAS ${ }^{\star \star \star \star \star} 7,8,40$ e o estudo de prevalência. Dois aspectos já abordados em trabalhos anteriores 42,43 merecem ser retomados no presente artigo. O primeiro refere-se ao fato de o estudo de validação e confiabilidade ter sido realizado apenas em Copa-

* Ver periodo e demais detalhes no trabalho de Veras ${ }^{47}$ (1992).

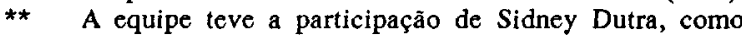
supervisor de campo, e dos seguintes trabalhadores de campo: Cristina Souza, Fátima Ventura, Rosane Milioli, Angélica Ferreira, Sergio Lyra e Eduardo Valarin. Para a validação do questionário, dois psiquiatras estiveram presentes: Evandro Coutinho (urn dos autores do artigo) e George Ney Jr.

$\star \star \star$ Maiores detalhes podem ser obtidos em trabalho de um dos autores deste artigo (Veras ${ }^{47}, 1992$, pp. 108 a 112).

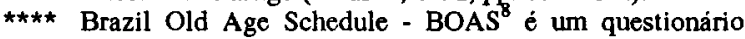
multidimensional de avaliação funcional, desenvolvido para estudos com a população idosa de um centro urbano (Rio de Janeiro), baseado em outros instrumentos que obtiveram padrōes aceitáveis de validade e confiabilidade, como o questionário CARE ${ }^{16}$, para o segmento de saúde mental, e o OARS $^{14}$ e PAHO ${ }^{33}$.

O questionário multidimensional visa a cobrir as áreas consideradas mais importantes da vida do idoso, a saber: saúde física, saúde mental, condição social, condição econômica e atividade do dia-a-dia

$\star \star \star \star \star$ Veja esta discussão em artigos anteriores ${ }^{42,43}$. cabana. Outro aspecto do estudo a ser retomado refere-se aos critérios utilizados para a pontuação das respostas do segmento de saúde mental do questionário BOAS. No estudo, o questionário utilizado é uma tradução adaptada para o português, do instrumento $C A R E$, utilizado em vários estudos na Inglaterra, principalmente pelo grupo de pesquisadores de Liverpool e do Guy's Hospital de Londres $^{12,17,26,27}$.

Apesar de todos os trabalhadores de campo estarem muito bem treinados e em perfeita harmonia, pudemos identificar problema em um item para o diagnóstico de síndrome cerebral orgânica ${ }^{* \star \star \star \star *}$. A interpretação dada pela equipe e a conseqüente marcação de pontos não estava em sintonia com a interpretação dos trabalhos dos pesquisadores ingleses. Apesar de as mudanças serem mínimas, e as alterações dos resultados exíguas, para uma transparência nos trabalhos científicos foram apresentados os resultados retificados, pois reconhece-se serem essas as marcações de pontos mais adequadas.

Os valores de sensibilidade, especificidade e os valores Kappa para os estudos de validação e confiabilidade definiram o ponto de corte $2 / 3$ para sindrome cerebral orgânica (SCO). Apesar do problema de pontuação já mencionado, este não foi suficiente para alterar o ponto de corte, o que confirma que o ajuste nos critérios de julgamento e conseqüente pontuação de "suspeição de casos" foi pequeno. Este ponto de corte, aliás, é o mesmo usado no estudo do Guy's/Age Concern ${ }^{26,27}$, confirmando a similaridade nas versões dos instrumentos.

A Tabela 2 apresenta os índices do estudo de validação, usando os pontos selecionados para SCO.

No presente artigo, algumas vezes aparece a categoria demência. $\mathrm{Na}$ verdade, esse uso não é o mais apropriado. $O$ questionário BOAS detecta apenas as deficiências e/ou debilidade cognitiva, que é um indicador ou estágio inicial da maioria dos quadros demenciais. Como em alguns trabalhos faz-se uso indiscriminado de sindrome cerebral orgânica e debilidade cognitiva (na literatura inglesa, organic brain syndrome e cognitive impairment, respectivamente) para se referir aos achados de "suspeição de casos" de debilidade cognitiva, considerou-se prudente esta advertência. Portanto, em várias passagens do presente texto aparece referência à demência ou $\mathrm{SCO}$ quando de fato está designando cognitive impairment. 
Tabela 2: Validação do ponto de corte do BOAS para Sindrome Cerebral Orgânica(SCO)

\begin{tabular}{|c|c|c|c|c|c|c|}
\hline & & \multicolumn{3}{|c|}{ sco } & sensibilidade & $100 \%$ \\
\hline & & + & - & & especifidade & $90,7 \%$ \\
\hline \multirow{3}{*}{$\begin{array}{l}\text { BOAS } \\
\text { BOAS }\end{array}$} & + & 5 & 4 & 09 & valor preditivo positivo & $55,6 \%$ \\
\hline & - & 0 & 39 & 39 & valor preditivo negativo & $100 \%$ \\
\hline & & 05 & 43 & 48 & erro de classificação & $8,3 \%$ \\
\hline
\end{tabular}

Tabela 3: Indicadores demográficos da amostra por sexo e distrito: faixa etária e nivel de instrução.

\begin{tabular}{|c|c|c|c|c|c|c|}
\hline \multirow[b]{2}{*}{ Indicadores } & \multicolumn{2}{|c|}{ Copacabana } & \multicolumn{2}{|c|}{ Méier } & \multicolumn{2}{|c|}{ S.Cruz } \\
\hline & Masc. & Fem. & Masc. & Fem. & Masc. & Fem. \\
\hline de 60 a 69 anos & $33(35,9 \%)$ & $79(49,4 \%)$ & $46(48,0 \%)$ & $69(46,6 \%)$ & $56(58,3 \%)$ & $78(53,4 \%)$ \\
\hline de 70 a 79 anos & $40(43,5 \%)$ & $59(36,9 \%)$ & $37(38,5 \%)$ & $53(35,8 \%)$ & $34(35,4 \%)$ & $54(37,0 \%)$ \\
\hline 80 ou + anos & $19(20,6 \%)$ & $22(17,7 \%)$ & $13(13,5 \%)$ & $25(16,9 \%)$ & $6(6,3 \%)$ & $12(8,2 \%)$ \\
\hline Total & $92(100 \%)$ & $160(100 \%)$ & $96(100 \%)$ & $147(99,3 \%)^{(a)}$ & $96(100 \%)$ & $144(98,6 \%)^{(b)}$ \\
\hline sem instrução & $2(2,2 \%)$ & $14(8,8 \%)$ & $28(29,2 \%)$ & $62(41,9 \%)$ & $52(54,2 \%)$ & $98(67,1 \%)$ \\
\hline primeiro grau & $8(8,7 \%)$ & $22(13,8 \%)$ & $24(25,0 \%)$ & $42(28,4 \%)$ & $21(21,9 \%)$ & $30(20,6 \%)$ \\
\hline segundo grau & $37(40,7 \%)$ & $89(55,6 \%)$ & $39(40,6 \%)$ & $39(26,4 \%)$ & $18(18,7 \%)$ & $15(10,3 \%)$ \\
\hline superior & $43(46,2 \%)$ & $28(17,5 \%)$ & $3(3,1 \%)$ & $2(1,3 \%)$ & $3(3,1 \%)$ & $0(0,0 \%)$ \\
\hline Total & $90(97,8 \%)^{(0)}$ & $153(95,6 \%)^{(d)}$ & $94(97,9 \%)^{(\theta)}$ & $145(98,0 \%)^{(n)}$ & $94(97,9 \%)^{(g)}$ & $143(98,0 \%)^{(h)}$ \\
\hline
\end{tabular}

Nota - Há um dado de informação faltando na coluna (a), e dois na coluna (b). Em $(c, d, e, f, g, h)$ há 2, 7, 2, 3, 2 e 3 informaçōes faltando, respectivamente.

\section{Resultados}

A fim de determinar se as diferenças entre os três bairros resultavam de constituições diferenciadas dessas populações em relação à faixa etária e ao sexo, a população de cada um deles foi estratificada, e as proporções de deficiência cognitiva para homens e mulheres foram calculadas em separado.

Na Tabela 3, encontra-se a distribuição das amostras por distritos, segundo sexo, faixa etária e escolaridade. Observa-se que a população de Copacabana tem os individuos mais velhos e com melhor nivel de escolaridade, enquanto Santa Cruz situa-se no extremo oposto. Nessa tabela, nos distritos do Méier e Santa Cruz foram perdidas uma e duas informações, respectivamente, relativas a mulheres idosas que não forneceram a idade. No entanto, segundo avaliação dos entrevistadores, esses três casos são de idosos com idade provavelmente acima de 80 anos. Por isso, quando da análise dos resultados, esses idosos serão incluídos no grupo de 80 anos ou mais.

\section{Deficiência cognitiva (SCO)}

Na Tabela 4 são apresentados os resultados da prevalência de SCO para cada distrito. Na Tabela 5 encontra-se a distribuição dos entrevistados segundo o escore obtido para SCO. Um escore mais elevado indica sintomas com maior freqüência ou severidade.

Em Copacabana, numa amostra de 252 pessoas foi definido um total de 6 homens e 9 mulheres, pelo BOAS, como sofrendo de sintomas de deficiência

Tabela 4. Taxas de prevalência de Síndrome Cerebral Orgânica $(\mathrm{SCO})$ por distritos, segundo o BOAS (os valores entre parênteses apresentam intervalo de confiança de 95\%).

\begin{tabular}{cccc}
\hline & $\begin{array}{c}\text { Copacabana } \\
(n=252)\end{array}$ & $\begin{array}{c}\text { Méier } \\
(n=244)\end{array}$ & $\begin{array}{c}\text { S.Cruz } \\
(n=242)\end{array}$ \\
\hline SCO & $5,9( \pm 2,92)$ & $9,8( \pm 3,74)$ & $29,7( \pm 5,76)$ \\
\hline
\end{tabular}


Tabela 5. Distribuição de freqüència e percentagem da escala de síndrome Cerebral Orgânica(SCO) por distrito.

\begin{tabular}{|c|c|c|c|c|c|c|}
\hline \multirow[t]{2}{*}{ Escore } & \multicolumn{2}{|c|}{$\begin{array}{c}\text { Copacabana } \\
N=252\end{array}$} & \multicolumn{2}{|c|}{$\begin{array}{c}\text { Méier } \\
N=244\end{array}$} & \multicolumn{2}{|c|}{$\begin{array}{l}\text { S.Cruz } \\
N=242\end{array}$} \\
\hline & Freq. & $\%$ & Freq. & $\%$ & Freq. & $\%$ \\
\hline 0 & 158 & 62,7 & 96 & 39,3 & 56 & 23,1 \\
\hline 1 & 69 & 90,1 & 103 & 81,6 & 76 & 54,5 \\
\hline 2 & 10 & 94,0 & 21 & 90,2 & 38 & 70,2 \\
\hline 3 & 5 & 96,0 & 8 & 93,4 & 24 & 80,2 \\
\hline 4 & 5 & 98,0 & 4 & 95,1 & 24 & 90,1 \\
\hline 5 & 0 & 98,0 & 4 & 96,7 & 14 & 95,9 \\
\hline 6 & 4 & 99,6 & 4 & 98,4 & 7 & 98,8 \\
\hline 7 & 1 & 100,0 & 4 & 100,0 & 2 & 99,6 \\
\hline 8 & 0 & 100,0 & 0 & 100,0 & 1 & 100,0 \\
\hline
\end{tabular}

Tabela 6. Prevalência de SCO, de acordo com o BOAS, por faixa etária e sexo, nos três distritos estudados.

\begin{tabular}{|c|c|c|c|c|c|c|}
\hline \multirow[b]{2}{*}{ Idade } & \multicolumn{2}{|c|}{ Copacabana } & \multicolumn{2}{|c|}{ Méier } & \multicolumn{2}{|c|}{ Santa Cruz } \\
\hline & Masc. & Fem. & Masc. & Fem. & Masc. & Fem. \\
\hline $60-69$ & $0.0 \%$ & $0.0 \%$ & $2.2 \%$ & $7.2 \%$ & $21.4 \%$ & $25.6 \%$ \\
\hline $70-79$ & $7.5 \%$ & $5 \%$ & $2.7 \%$ & $13.2 \%$ & $20 \%$ & $35 \%$ \\
\hline $80 e+$ & $15 \%$ & $27 \%$ & $7 \%$ & $34.6 \%$ & $83.3 \%$ & $64 \%$ \\
\hline Total & $6.5 \%$ & $5.6 \%$ & $3.1 \%$ & $14.2 \%$ & $25.0 \%$ & $33 \%$ \\
\hline
\end{tabular}

Nota - No grupo de mulheres idosas, nos distritos do Méier e Santa Cruz, grupo etário de 80 anos e mais, estão incluidos os três idosos da categoria "informação ignorada" (ver Tabela 3), pelos motivos já apresentados.

cognitiva relacionada à $\mathrm{SCO}$. A percentagem foi de $5,9 \%$ do total da amostra, $6,5 \%$ para os homens e $5,6 \%$ para as mulheres. A diferença entre os sexos não foi significativa estatisticamente $(P=0,772)$. A Tabela 6 apresentam-se os casos de deficiência cognitiva por sexo e faixa etária, verificando-se um número crescente de SCO com o avanço da idade, em particular na faixa etária acima de 80 anos.

No Méier, entre 242 idosos, um total de 3 homens e 21 mulheres foi definido pelo BOAS como de pessoas que sofriam de SCO. A percentagem foi de $9,8 \%$, considerando o total da amostra, $3,1 \%$ dos homens e $14,2 \%$ das mulheres. A diferença entre os sexos é altamente significativa em termos estatisticos $(P=0,005)$. Os casos de deficiência cognitiva por sexo e faixa etária estão na Tabela 6 , onde se observa que um número crescente de pessoas apresenta deficiência com $\circ$ aumento da idade.

Em Santa Cruz, um total de 24 homens e 48 mulheres, numa amostra de 242 , foi considerado pelo BOAS como de pessoas que sofriam de SCO. A percentagem foi de $29,7 \%$ do total da amostra, 25,0\% para os homens e $32,9 \%$ para as mulheres. Apesar da predominância de casos entre as mulheres, não existe diferença significativa em termos estatísticos $(P=0,190)$. Na Tabela 6 apresenta-se a prevalência de deficiência cognitiva por sexo e faixa etária, demonstrando, como nos outros distritos, um aumento com a idade.

\section{Discussão dos resultados de prevalência}

Os resultados podem ser resumidos na afirmação de que a taxa de deficiência cognitiva de Copa- 
cabana e do Méier foram muito próximas, considerando o grande intervalo de confiança, ao passo que Santa Cruz apresentou uma prevalência muito mais alta. Quando comparados a estudos em outros países, utilizando metodologia semelhante, os resultados de Copacabana e do Méier estão próximos à média. No entanto, os resultados para Santa Cruz são extremamente altos.

Deve-se frisar que as amostras não são representativas da cidade como um todo. Os resultados se referem apenas aos distritos estudados, e não à cidade do Rio de Janeiro.

Um grande número de levantamentos da prevalência de demência já foi realizado em várias partes do mundo, sobretudo Europa, América do Norte e Japão. As taxas relatadas possuem variação ampla. Essas variações são devidas principalmente às diferenças de abordagem, tais como critérios distintos para a avaliação da presença ou ausência de sintomas e sinais; inquérito domiciliar ou institucional (pessoas já sob assistência); proporção elevada de idosos mais velhos no denominador populacional, entre outros fatores. Todavia, para os estudos que utilizaram metodologias semelhantes até recentemente, os levantamentos de pessoas idosas têm sido relativamente coincidentes no que diz respeito à determinação da taxa de prevalência de SCO de cerca de $7 \%$, tanto para os casos leves quanto para os graves, e de cerca de $20 \%$ para os que têm mais de 80 anos ${ }^{19,51}$. Estudos recentes sugerem que isto pode ser alto demais ${ }^{10,20,24,26,31}$, apontando para não mais de $4,5 \%$ de SCO total em levantamentos domiciliares de pessoas idosas. Foram registradas taxas de prevalência ainda mais baixas em alguns estudos cujos critérios para a definição dos casos eram mais exigentes ${ }^{21,24}$. Contudo, instrumentos curtos ('brief schedule', na literatura inglesa), tal como o utilizado no presente estudo, apenas detectam a deficiência cognitiva e podem superestimar a prevalência de demência uma vez que uma proporção dos casos será conseqüência de estados não-progressivos ou reversíveis, tais como deficiência mental, traumatismos cranianos, delírio ou distúrbios afetivos. As taxas muito baixas registradas nos estudos de prevalência recentes não foram obtidas em brief schedules ${ }^{26,28}$, mas sim com instrumentos de entrevistas mais completas.

Os estudos de incidência têm sido raros, mas uma investigação da população total de Lundby, Suécia, encontrou um declínio na incidência no decorrer de aproximadamente 25 anos para todos os tipos de demência ${ }^{18}$. A tendência descendente na prevalência da demência pode assim refletir uma mudança real na incidência ${ }^{3,26}$. Se a incidência de demência está diminuindo nos países mais desenvolvidos, caracteriza-se um efeito de coorte ou de geração. Variáveis associadas ao modo de vida, histórico empregatício e lazer podem ser relevantes. Sabe-se que vários fatores potencialmente passíveis de prevenção, que podem ter sido alterados entre as sucessivas coortes, influenciam no desenvolvimento da demência, particularmente na etiologia da demência traumática e cerebrovascular ${ }^{3}$.

No presente estudo, as taxas de deficiência cognitiva situaram-se ao longo de um espectro amplo, sendo aproximadamente a média de outros estudos em Copacabana $(5,9 \%)$, acima da média no Mëier $(9,8 \%)$ e muito mais altas em Santa Cruz $(29,7 \%)$. É difícil explicar esta discrepância através de problemas metodológicos, pois a mesma metodologia foi aplicada nas três áreas. Estas taxas tornam-se ainda mais inesperadas, se considerado o fato de que a faixa etária da amostra começava de 60 anos e não de 65 , como em muitos outros levantamentos da população idosa. A população idosa do bairro de Santa Cruz, onde as taxas foram as mais altas, é composta principalmente de pessoas idosas com menos de 70 anos. Jorm e col. ${ }^{21}$ concluíram que há aumento exponencial após a idade de 60 , e que a prevalência de demência é aproximadamente o dobro a cada faixa etária de cinco anos. Além disso, o presente estudo excluiu as populações institucionais, onde as taxas de demência são sabidamente muito mais altas.

Uma explicação possivel para esses níveis altos poderia ser a instrução e o nível socioeconômico da amostra. Gurland $^{17}$, em Nova York, Cooper e Bi$\mathrm{ckel}^{11}$, em Manheim, e mais recentemente, Weissman e col. ${ }^{49}$, em New Haven sugeriram que existe relação inversa entre status socioeconômico e a prevalência de demência. A influência dos níveis de instrução nos resultados dos levantamentos é sabidamente importante no Brasil e em muitos outros paises.

Nos países desenvolvidos, o analfabetismo, mesmo em pessoas idosas, é muito incomum, e os níveis socioeconômicos têm um padrão muito mais alto se comparados aos dos países do Terceiro Mun- 
do. No Brasil, existem poucos levantamentos domiciliares da população idosa, mas todos os bons estudos anteriores encontraram taxas muito altas de deficiência cognitiva e outros distúrbios mentais. Almeida Filho ${ }^{1}$, em um estudo na Bahia, obteve os seguintes resultados: $6,8 \%$ para a demência e $36,4 \%$ quando incluídas todas as sindromes de doença mental. Blay, em São Paulo, usando um FaceHand Test, um instrumento planejado para detectar a SCO na população idosa, encontrou uma prevalência de 5,5\% em uma amostra de sujeitos acima de 65 anos. Ramos ${ }^{34}$ encontrou uma taxa de prevalência de distúrbio psiquiátrico de $25 \%$ entre os idosos de São Paulo.

Mesmo nos estudos da população total de adultos de todas as idades, as taxas de prevalência têm sido muito altas no Brasil. Por exemplo, o estudo realizado em três clínicas de assistência básica em São Paulo ${ }^{29}, 30$ encontrou taxa de prevalência de distúrbio psiquiátrico que variava de $47 \%$ a $56 \%$. Mais recentemente, foi empreendido estudo baseado num levantamento domiciliar na favela da Rocinha, no Rio de Janeiro, abrangendo as mães que criavam crianças de menos de cinco anos. Este estudo apresentou uma taxa de prevalência de $36 \%$ para distúrbio psiquiátricos ${ }^{36}$.

Em Santa Cruz e, em menor grau no Méier, as amostras abrangiam pessoas de níveis de instrução mais baixos, tendo-se decidido examinar mais detidamente os itens nas escalas de SCO nas quais os entrevistados apresentavam índices positivos (ver Tabela no Anexo). A análise dos itens de índice alto revelou que muitos entrevistados compreendiam mal algumas perguntas, provavelmente em conseqüência de seu nível de instrução mais baixo e da pouca familiaridade com questionários. Portanto, uma explicação provável para as taxas de prevalência muito mais altas, particularmente as do bairro de Santa Cruz, é a possivel inadequação da escala para uso naquele universo cultural e social. Por exemplo, pode-se ver, no Anexo, que em Santa Cruz, 24,8\% não sabem o nome do presidente do Brasil, 23,9\% não sabem em que ano estão, e 66,9\% não se lembram do nome da instituição que estava realizando o levantamento. É possível que nessa área rural tais informações não sejam alvo da mesma importância que nas outras duas. Em contraste, no levantamento de Nova York/Londres ${ }^{17}$, apenas $4 \%$ e $6 \%$ dos idosos não sabiam o nome do presi- dente e do primeiro-ministro, respectivamente. Em um estudo realizado em São Paulo por Blay e col. ${ }^{6}$, usando o "Mental Status Questionnaire" (MSQ), os autores usaram uma análise discriminante a fim de selecionar os melhores itens do MSQ para identificar demência. As perguntas sobre o ano em curso e o nome do presidente apareceram como as perguntas mais discriminantes, do mesmo modo que no presente estudo. No entanto, o elevado grau de erro sugere que, no Brasil - particularmente entre os que têm pouca instrução -, esses itens podem fornecer informaçōes enganosas, talvez mais pelo baixo grau de escolaridade do que devido a uma "real" deficiência de memória.

Em relação ao nome da instituição que realizou o levantamento, muitas pessoas em Santa Cruz não conseguiam lembrar três palavras simples - Instituto de Medicina Social - embora tenham recebido uma carta da instituição pedindo permissão para a entrevista. Deve-se considerar que algumas pessoas não tenham lido a carta por serem analfabetas, e talvez que, no seu universo cultural, essas palavras não pertençam ao cotidiano, sendo por isso difíceis de lembrar.

Uma explicação alternativa a ser considerada é o nível mais alto de ansiedade vivido durante a entrevista por aqueles de menor instrução. Muitas pessoas afirmaram que haviam sido emocionalmente tocadas pela entrevista e agradeceram o entrevistador pela oportunidade de participarem do estudo, ou que pela primeira vez em muitos anos um "médico" dera atenção aos seus problemas. Comparando os três itens freqüentes em outros bairros, na área de Copacabana, apenas $7,1 \%$ não sabiam o nome do presidente, e $4 \%$ não sabiam em que ano estavam. O Méier está na posição intermediária. Contudo, o nome "Instituto de Medicina Social" foi considerado difícil de se lembrar por um terço dos respondentes de Copacabana e por metade da amostra de idosos do Méier.

A inadequação de certos outros itens da escala de SCO deve ser explorada em profundidade nos próximos estudos em áreas pobres do Rio de Janeiro. Considerando-se a hipótese de o nivel de instrução alterar significativamente a avaliação da $\mathrm{SCO}$, ainda que apenas para uma minoria de idosos, este problema metodológico contribuiria para um aumento artificial nas taxas de prevalência em estudos em regiōes pobres. 
Ainda que os problemas de método possam contribuir para as diferenças de prevalência, é possível que mudanças no modo de vida e no comportamento, relacionadas com o declínio na incidência de SCO, não estejam ocorrendo de forma homogênea no Brasil. Em outras palavras, as diferenças nas taxas de prevalência poderiam refletir diferenças reais na freqüência da condição. Talvez algumas destas "tendências de Primeiro Mundo" só se tornarão realidade, nos países do Terceito Mundo, no futuro, e, a princípio, as melhoras só serão detectadas nas áreas mais desenvolvidas. Esta hipótese merece maiores investigações. As taxas de prevalência mais baixas em Copacabana, quando comparadas com as de outros bairros, seriam compatíveis com a idéia de que o "efeito de coorte" atribuível a mudanças no modo de vida estivesse influenciando este bairro mais próspero, de características mais próximas aos países desenvolvidos, se comparado aos dois outros bairros. As mudanças de dieta, menor consumo de tabaco e álcool e a redução da exposição a poluentes ocupacionais e ambientais são caracteristicas de uma sociedade saudável e abastada. A persistência desses fatores ambientais pode ser maior nas áreas mais pobres.

Quando estratificados por sexo (Tabela 6), houve entre as mulheres maior prevalência de deficiência cognitiva, particularmente nos bairros do Méier e Santa Cruz. Mais uma vez, Copacabana apresentou um padrão diferente, onde os homens e mulheres não diferiam em suas taxas ajustadas segundo a idade. A diferença encontrada entre os sexos poderia novamente refletir os níveis socioeconômicos e de instrução mais baixos entre as mulheres, se comparados aos dos homens, nas áreas mais pobres, sugerindo que a explicação metodológica é correta.

Como em outros estudos, as taxas de deficiência cognitiva são significativamente afetadas pela idade. Isto ocorreu em todos os bairros. $\mathrm{A}$ amostra reflete a distribuição da população, e portanto a proporção de membros da faixa de "velhos velhos" (acima de 80 anos) não é grande. O número pequeno nesta faixa não permite um exame maior, mas em Copacabana, dentre os 15 casos, 9 pertenciam à faixa de "velhos velhos". Uma outra hipótese que poderia ser explorada em relação à taxa de prevalência mais alta no presente estudo é que, nos paises desenvolvidos, uma proporção importante da popu- lação idosa vive só em suas casas, em conseqüência de a família ser pequena. Por causa disso, há muito mais estabelecimentos residenciais institucionais, providos tanto pelo setor público quanto pelo privado, para apoiar as pessoas que têm necessidade disso, em comparação com os estabelecimentos residenciais existentes no Brasil. Nos paises desenvolvidos, cerca de $5 \%$ dos idosos vivem em instituiçōes ${ }^{2}$ e, dentre estes, estima-se que de $30 \%$ a $70 \%$ sofram de demência ${ }^{24}$. No Brasil, a proporção de pessoas idosas institucionalizadas é estimada em menos de $1 \%$. Quando se realiza um levantamento domiciliar em paises desenvolvidos, mesmo com não mais do que $5 \%$ vivendo em instituições, existe a possibilidade de que algumas pessoas sejam excluidas do estudo por serem institucionalizadas, 0 que exclui alguns "casos" da comunidade, dando uma taxa de prevalência mais baixa. Isso traz algumas dificuldades para a comparação de taxas com paises onde quase todas as pessoas idosas não estão em instituições e sim em casa, portanto, correndo o "risco" de serem aleatoriamente selecionadas para um estudo domiciliar.

O controle de muitas doenças potencialmente fatais melhorou nas últimas décadas e aumentou a expectativa de vida de muitas pessoas idosas. Mais pessoas estão atingindo um periodo da vida em que problemas circulatórios e outras doenças degenerativas se tornam manifestos. Uma vez que as taxas de prevalência são determinadas tanto pela incidência quanto pela duração do tempo de vida, a sobrevivência após a ocorrência de demência precisa ser considerada ao se interpretarem as taxas de prevalência. Isso é particularmente importante quando se comparam dados de países desenvolvidos com os menos desenvolvidos. Por exemplo, sabe-se que existem padrões diferentes de expectativa de vida ao nascimento e à idade de 60 anos ${ }^{37}$. Na Europa, a Grécia, a despeito de não ser o país mais rico da região, tem a expectativa de vida mais alta na idade de $60 \operatorname{anos}^{52}$. Na América do Norte, é surpreendente constatar que as mulheres mexicanas com 65 anos têm em média mais três anos de vida do que as mulheres do vizinho Estados Unidos ${ }^{22}$. De modo semelhante, quando comparado com os britânicos, o brasileiro tem uma maior expectativa de vida aos $60 \operatorname{anos}^{35}$. A razão disso é que os "pobres" que chegam vivos até os 60 anos constituem uma amostra altamente selecionada de sobreviventes. Prova- 
velmente os que sobrevivem até a velhice no Méier e em Santa Cruz constituem um grupo fisicamente saudável que tem um tempo de vida aumentado. Contudo, esta longevidade ampliada não significa que eles estejam necessariamente livres de doenças mentais.

Se esta hipótese estiver correta - de que o tempo de vida aumentado está associado a um aumento dos distúrbios mentais -, pode-se esperar que a taxa de prevalência de demência na velhice aumente mais rápido ${ }^{15} \mathrm{em}$ países menos desenvolvidos, em contraste com os resultados recentes em paises desenvolvidos.

Em quase todos os estudos foi demonstrada uma correlação entre deficiência cognitiva e nível socioeconômico, juntamente com o nivel de instrução. A fim de suplantar este viés cultural da influência da instrução e da classe social nos índices desse tipo de escala, foi sugerido que diferentes pontos de corte deveriam ser usados, ou que deveriam ser feitos ajustes usando uma equação de regressão ${ }^{25}$. Em um estudo recente, num país menos desenvolvido, sugeriu-se um ponto de corte diferente, a fim de fazer frente a esse problema ${ }^{52}$. Todavia, existem dúvidas consideráveis em relação à utilidade desses ajustes, uma vez que eles provavelmente levarão a erro se empregados em populações amplamente diferenciadas ${ }^{9,32}$. Uma vez que o estudo de validação não foi realizado no bairro de Santa Cruz, esse ajuste não foi testado no nosso estudo. Como não existem diferenças metodológicas entre os bairros, as hipóteses enfocadas acima, tais como "efeito de coorte", duração da sobrevivência após a ocorrência de demência e a proporção mais baixa de pessoas idosas institucionalizadas, têm que ser consideradas. Contudo, as imperfeições metodológicas resultantes da inadequação de algumas perguntas do questionário BOAS, para os que têm nível sócioeconômico e de instrução menor, podem ter exercido influência muito importante na produção desses resultados.

\section{Atividade Diária e Sindrome Cerebral Orgânica}

Nos questionários multidimensionais para idosos, existe uma seção intitulada atividades diárias, ADL na literatura inglesa (activity daily living), que se constitui num indicador muito sensivel na definição da qualidade de vida do idoso e de grande valor para o planejamento de saúde.

No questionário BOAS, na seção atividades diárias, há uma pergunta formada de quinze itens concernentes à capacidade de desempenho das atividades diárias. $\mathrm{Na}$ análise das respostas dessa pergunta, os entrevistados foram divididos em dois grupos: os que informaram serem capazes e os sem condições de executar a tarefa*.

As questões foram analisadas uma a uma, e, a partir do conjunto de suas freqüências, esses itens foram ordenados em uma escala e divididos em quatro grupos, isto é, itens com freqüências similares foram agrupados. Em nosso estudo, a escala das atividades, da de mais fácil desempenho para a mais difícil, é a seguinte:

nivel um de dificuldade 1. alimentar-se sozinho, 2. pentear o cabelo, 3. deitar-se e levantar-se da cama, 4 . vestir-se.

nivel dois de dificuldade 5. tomar banho, 6. caminhar em superfície plana, 7. usar o banheiro a tempo, 8. tomar remédios.

nivel três de dificuldade 9. sair para perto de casa, 10. subir escadas.

nivel quatro de dificuldade 11. cortar as unhas dos pés, 12. sair para distâncias longas, 13. tomar ônibus.

Como descrito nos demais trabalhos, este estudo confirmou os achados anteriores que demonstram que os mais idosos e as mulheres são os que apresentam mais dificuldades para o desempenho das atividades do dia-a-dia, apesar de a capacidade de desempenho da atividade diária estar preservada na imensa maioria dos idosos (Tabela 7). Assim como no estudo de saúde mental, os residentes nas regiões mais pobres tiveram um pior desempenho. Os resultados mostraram que $30,9 \%$ dos entrevistados $(18,4 \%$ no sexo masculino e $39 \%$ no sexo feminino) do grupo etário mais idoso relataram problema no desempenho das atividades considera-

A descrição detalhada da metodologia empregada e maiores informações podem ser obtidas em trabalho de Veras ${ }^{47}$ (1992). 
Tabela 7. Percentual de idosos, por sexo e grupo etário, que conseguem executar um conjunto de atividades diárias, considerando-se quatro niveis de dificuldades.

\begin{tabular}{|c|c|c|c|}
\hline \multirow[b]{2}{*}{$\begin{array}{l}\text { Atividades diárias } \\
\text { grupo }\end{array}$} & \multicolumn{3}{|c|}{ Masculino } \\
\hline & $\begin{array}{l}60-69 \\
N=135\end{array}$ & $\begin{array}{l}70-79 \\
N=111\end{array}$ & $\begin{array}{l}80 \text { or }+ \\
N=38\end{array}$ \\
\hline nivel 1 & $0,74 \%$ & $0 \%$ & $2,63 \%$ \\
\hline nivel 1 e 2 & $3,70 \%$ & $1,80 \%$ & $2,63 \%$ \\
\hline nivel 1,2 e 3 & $3,70 \%$ & $9,01 \%$ & $13,16 \%$ \\
\hline \multirow[t]{2}{*}{ nivel $1,2,3$ e 4} & $91,85 \%$ & $89,19 \%$ & $81,58 \%$ \\
\hline & \multicolumn{3}{|c|}{ Feminino } \\
\hline Atividades diárias & $60-69$ & $70-79$ & 80 or + \\
\hline grupo & $N=226$ & $N=166$ & $N=59$ \\
\hline nivel 1 & $0,44 \%$ & $2,41 \%$ & $6,78 \%$ \\
\hline nível 1 e $2^{(a)}$ & $1,33 \%$ & $3,61 \%$ & $11,86 \%$ \\
\hline nivel 1,2 e 3 & $7,96 \%$ & $17,47 \%$ & $20,34 \%$ \\
\hline nivel $1,2,3$ e $4^{(b)}$ & $90,27 \%$ & $76,51 \%$ & $61,02 \%$ \\
\hline
\end{tabular}

Nota- (a) Existe uma perda de informações no nivel 2; e (b) existem duas perdas no nivel 4.

das mais difíceis (nivel 4), enquanto nos idosos jovens (60 a 69 anos) a proporção para igual dificuldade cai para $9,1 \%(8,1 \%$ no sexo masculino e $9,7 \%$ no sexo feminino). Entre aqueles com mais de 80 anos, $5,1 \%$ (2.6\% dos homens e $6,8 \%$ das mulheres) não conseguiram desempenhar tarefas mais simples, enquanto entre os idosos do grupo etário compreendido entre 60 e 69 anos este percentual caiu para $0,5 \%(0,7 \%$ dos homens e $0,4 \%$ das mulheres). Apesar de pequeno, o grupo nivel 1 é constituído de idosos totalmente dependentes e que precisam de ajuda constante para todas as atividades do dia-a-dia, até as mais simples.

Para se operacionalizar o indicador atividades diárias (AD) na associação com os resultados de SCO consideram-se autônomos todos aqueles em perfeita autonomia ou com dificuldades leves e/ou compativeis com a idade (niveis 3 e 4), incluindo no grupo dos sem autonomia apenas os que não conseguem desempenhar as tarefas dos níveis de dificuldade 1 e 2.

Observou-se uma importante associação entre debilidade cognitiva e AD. Aqueles com incapacidade de desempenhar atividades diárias nos níveis 1 e 2 são os mais prováveis de terem deficiência cognitiva. No entanto, devido aos problemas mencionados sobre a informação de SCO e pelo pequeno número de idosos nos níveis 1 e 2 , as razões de prevalência observadas serão tratadas apenas como um indicativo a merecer confirmação em um próximo estudo. Com as reservas assinaladas, verificou-se que aqueles com mais dificuldade no desempenho das atividades diárias (nivel 1), ou seja, o grupo mais dependente, mostram maior prevalencia de doença. A prevalência no nível 1 é cerca de duas vezes maior do que no nivel 2 , e cinco vezes maior do que nos autônomos.

\section{Considerações Finais}

O desenvolvimento de novos indicadores e/ou novas abordagens visa, principalmente, a propiciar instrumentos eficazes no apoio à detecção precoce de muitos dos problemas que hoje acometem a população idosa.

Essa divisão da cidade permitiu observar que apesar da maior prevalência na região pobre, sua população é constituída de idosos mais jovens do que na área rica. Este fato leva a supor que os casos de demência, na grande maioria, se constituem de debilidade cognitiva em estágio inicial. A fim de se adiar, pelo maior número de anos o avançar dessa doença crônica, as medidas de planejamento e de serviços de saúde devem se dar, prioritariamente, no plano do cuidado primário, através de atividades que contem com visitadoras domiciliares, atividades sociais, aconselhamento, acompanhamento e postos de saúde com pessoal treinado. Já a região mais rica tem a população constituída de idosos mais velhos, e, apesar de as taxas de prevalência serem inferiores, pessoas detectadas como casos são idosos em quem a debilidade cognitiva está mais avançada e os cuidados necessários são aqueles que requerem médicos especializados, medicamentos, residências para idosos e outros. Portanto, é preciso uma abordagem distinta, tanto do ponto de vista da organização dos serviços de saúde e treinamento de pessoal quanto dos recursos financeiros a serem despendidos.

A apresentação desse exemplo visa a reforçar a necessidade de estudos que levem em consideração as diferenças sociais, pois só desse modo é possivel detectar proporções de doenças tão distintas em uma mesma cidade, e do mesmo modo 
demandas de cuidados tão particulares, devido ao perfil social e demográfico das populações.

O diagnóstico e o tratamento de problemas de saúde nos idosos são particularmente difíceis por causa da variabilidade e da cronicidade de muitas doenças, e também devido à multiplicidade de sintomas. Além disso, entre as pessoas idosas, os problemas de saúde físicos e mentais são altamente correlacionados e podem ser interativos. Para os planejadores de políticas, o propósito dos levantamentos psiquiátricos é planejar instalaçōes e serviços para a população alvo. Os levantamentos são utilíssimos se fornecerem informações vinculadas aos aspectos relevantes da vida da pessoa idosa, tais como deficiência física, relações sociais, atividades da vida cotidiana, renda, estrutura familiar e assim por diante. Isso é de importância fundamental no Brasil, onde existem, presentemente, poucos estudos sobre $\mathrm{SCO}$ e a prevalência para este estado mórbido, na comunidade, mostrou-se alta, particularmente nas áreas pobres.

VERAS, R.P. \& COUTINHO, E. [Prevalence of organic brain syndrome in an elderly population in a metropolitan population of southeastem Brazil]. Rev. Saúde Pública, 28: 26-37, 1994. This paper is a summary of the main findings concerning organic brain syndrome (O.S.S.) in a prevalence study carried out among the elderly population of the city of Rio de Janeiro, Brazil. The project selected three districts of the city - Copacabana, Méier and Santa Cruz - in view of a set of indicators. The study for the validation and reliability of the diagnostic instrument was carried out in the district of Copacabana. Prevelence results for organic brain syndrome were $5.9 \%$ in Copacabana, $9.84 \%$ in Méier and $29,75 \%$ in Santa Cruz. Several hypotheses have been formulated in the attempt to explain such diverse results within a single city. Associations with a variety of socio-economic indicators are presented. Dependence and loss of autonomy in elderly people are closely associated with organic brain syndrome. The results are compared with national and international findings.

Keywords: Organic mental disorders, epidemiology. Depression, epidemiology. Aged. Questionnaires.

\section{Referências Bibliográficas}

1. ALMEIDA FILHO, N.; SANTANA, V.S.; PINHO, A.R. Estudo epidemiológico dos transtornos mentais em uma população de idosos: área urbana de Salvador-BA. J.Bras.Psiquiatr., 33:114-20, 1984.

2. AMES, D. Epidemiological studies of depression among the elderly in residential and nursing homes. Int.J.Geriatr.Psychiatry, 6:347-54, 1991.

3. ARIE, T. Questions in the psychiatry of old age. In: CBA
Foundation. Research and the ageing population. Chichester, 1988. Jonh Wiley \& Sons Ltd, (Ciba Foundation Syposium, 134). p. 86-105.

4. BERQUÓ, E.S. \& LEITE, V.M. Algumas considerações sobre a demografia da população idosa no Brasil. Ci.Cult., 40:679-88, 1988.

5. BLAY, S.L.; MARI, J.J.; RAMOS, L.R. The use of the facehand test to screen for organic brain syndromes: a pilot study. Rev. Saúde Pública, 23:395-400, 1989.

6. BLAY, S.L.; MARI, J.; RAMOS, L.R.; FERRAZ, M.P. Validity of the Brazilian version of the Mental Status Questionnaire (MSQ) as a screening test for dementia among elderly urban subjects: a pilot study. Int.J.Geriatr. Psychiatry, 6:779-85, 1991.

7. BOAS (Brazil Old Age Schedule) Manual. Instituto de Medicina Social, UERJ, 1987. Rio de Janeiro.

8. BOAS (Brazil old Age Schedule) Questionnaire - Multidimensional questionnaire for the elderly population. Instituto de Medicina Social, UERJ (University of the State of Rio de Janeiro), 1987. Rio de Janeiro.

9. BRAYNE, C. \& CALLOWAY, P. The association of education and socieconomic status with the mini mental state examination and the clinical diagnosis of dementia elderly people. Age Ageing, 19:91-6, 1990.

10. CLARKE, M.; LOWRY, R.; CLARKE, S. Cognitive impairment in the elderly: a community survey. Age Ageing, 15:278-84, 1986.

11. COOPER, B. \& BICKEL, H. Population screening and the early detection of dementing disorders in old age: a review. Psychol. Med., 14:81-95, 1984.

12. COPELAND, J.R.M.; GURLAND, B.J.; DEWEY,M.E.; KELLEHER, M.J.; SMITH, A.M.; DAVIDSON, I.A. Is there more dementia, depression and neurosis in New York? A comparative study of the elderly in New York and London using the computer diagnosis AGECAT. Br.J.Psychiatry, 151:466-73, 1987.

13. DORA, C. The determinants of chronic disease mortalities in old age: a follow up study in Porto Alegre, Brazil. London, 1992. [PhD thesis - London School of Hygiene and Tropical Medicine].

14. DUKE UNIVERSITY OARS. Multidimensional functional assessment: the OARS methodology. 2nd ed., Durham N.C., Duke University - Centre for the Study of Ageing and Human Development, 1978.

15. FRIES, J.F. \& CRAPO, L.M. Vitality and aging: implications of the rectangular curve. San Francisco, WH Freeman and Company, 1981.

16. GURLAND, B.J.; KURIANSKY, J.; SHARPE, L.; SIMON, R.; STILLER, P.; BIRKETT, P. The comprehensive assessment and referral evaluation (CARE): rationale, development and reliability. Int.J.Aging Hum.Dev, 8:9-42, 1977.

17. GURLAND, B.J.; COPELAND, J.R.M.; KELLEHER, M.J.; KURIANSKY, J.; SHARPE, L.; DEAN, L. The mind and mood of aging: mental health problems of the community elderly in New York and London. New York, Haworth Press, 1983.

18. HAGNELL, O.; LANKE, J.; RORSMAN, B.; OHMAN, R.; OJESJO, L. Current trends in the incidence of senile and multi-infarct dementia. Arch. Psychiatry Neurol. Sci., 233:423-38, 1983.

19. HENDERSON, A.S. \& KAY, D.W.K. The epidemology of mental disorders in the aged. In: Handbook of studies on psychiatry and old age. Oxford, Elsevier Science Publishers B.V., 1984. p.53-87.

20. INEICHEN, B. Measuring the rising tide. How many dementia cases will there be by 2001? Br.J.Psychiatry, 150:193-200, 
21. JORM, A.F.; KORTEN, A.E; HENDERSON, A.S. The prevalence of dementia: a quantitative integration of the literature. Acta Psychiatr. Scand, 76:465-79, 1987.

22. KALACHE, A.; VERAS, R.P.; RAMOS, L.R. O envelhecimento da população mundial: um desafio novo. Rev. Saúde Pública, 21:200-10, 1987.

23. KALACHE, A. Ageing in developing countries. In: Principles and practice of geriatric medicine. Chichester, John Wiley \& Sons Ltd., 1991. p.1517-28.

24. KAY, D.W.K. The epidemiology of dementia: a review of recent work Rev. Clin Gerontol., 1:55-66, 1991.

25. KITTNER, S.J.; WHITE, L.R.; FARMER, M.E.; WOLZ, M.; KAPLAN, E.; MOES, E.; BRODY, J.A.; FEINLEIB, M.F. Methodological issues in screening for dementia: the problem of education adjustment. J.Chronic. Dis., 39:163-70, 1986.

26. LINDESAY, J.; BRIGGS, K.; MURPHY, E. The guy's/age concern survey: prevalence rates of cognitive impairment, depression and anxiety in an urban elderly community. Br.J.Psychiatry, 155:317-29, 1989.

27. LINDESAY, J. The guy's/age concen survey: physical health and psychiatric disorder in an urban elderly community. Int.J.Geriatr. Psychiatry, 5:171-8, 1990.

28. LIVINGSTON, G.; HAWKINS, A.; GRAHAM, N.; BLIZARD, B.; MANN, A. The Gospel Oak Study: prevalence rates of dementia, depression and activity limitation among elderly residents in inner London. Psychol. Med., 20:137-46, 1990

29. MARI, J.J. Psychiatry morbidity in three primary medial care clinics in the city of São Paulo: issues on the mental health of the urban poor. Soc. Psychiatry, 22:129-38, 1987.

30. MARI, J.J.; IACOPONI, E.; WILLIAMS, P.; SIMŌES, O.; SILVA, J. B.T. Detection of psychiatric morbidity in the primary medical care setting in Brazil. Rev. Saúde Pública, 21:501-7, 1987.

31. MORGAN, K.; DALLOSO, H.M.; ARIE, T.; BYRNE, E.J.; JONES, R.; WAITE, J. Mental health and psychological well-being among the old and very old living at home. Br.J.Psychiatry, 150:801-7, 1987.

32. O'CONNOR, D.W.; POLLITT, P.A.; TREASURE, F.P.; BROOK, C.P.B.; REISS, B. The influence of education, social class and sex on Mini-Mental State scores. Psychol.Med., 19:771-6, 1989.

33. PAN AMERICAN HEALTH ORGANIZATION. Questionnaire for the elderly: epidemiology of ageing in Latin America and Caribbean. Washington, 1985.

34. RAMOS, L.R. Growing old in São Paulo, Brazil: assessment of health status and social support of elderly people from different socio-economic strata living in the community. London, 1986. [PhD thesis- London School of Hygiene and Tropical Medicine].

35. RAMOS, L.R.; VERAS, R.P.; KALACHE, A. Envelhecimento populacional: una realidade brasileira. Rev. Saúde Pública, 21:211-24, 1987.

36. REICHENHEIM, M.E. \& HARPHAM, T. Matemal mental health in a squatter settlement in Rio de Janeiro. Br.J.Psychiatry, 159:683-90, 1991.

37. SIEGEL, J.S. \& HOOVER, S.L. Demographic aspects of the health of the elderly to the year 2000 and beyond. World Health Stat.Q. , 32(314):133-202, 1982.

38. VERAS, R.P.; RAMOS, L.R.; KALACHE, A. Crescimento da população idosa no Brasil: transformaçōes e conseqüências na sociedade. Rev. Saúde Pública, 21:225-33, 1987.
39. VERAS, R.P. Considerações acerca de um jovem pais que envelhece. Cad Saúde Pública, 4:382-97, 1988.

40. VERAS, R.P.; SOUZA, C.A.M.; CARDOSO, R.F.; MILIOLI, R.; DUTRA, S. Pesquisando populações idosas: a importância do instrumento e o treinamento de equipe: uma contribuição metodológica. Rev.Saúde Pública, 22:513-8, 1988.

41. VERAS, R.P.; DUTRA, S.; SOUZA, C.A.M.; MILIOLI, R.; VENTURA, F. PToposta metodológica para inquérito domiciliar com populaçōes idosas em um centro urbano do Estado do Rio de Janeiro (Brasil). Rev. Saúde Pública, 23:429-38, 1989.

42. VERAS, R.P.; COUTINHO, E.; NERY JR., G. População idosa no Rio de Janeiro (Brasil): estudo-piloto da confiabilidade e validação do segmento de saúde mental do questionário BOAS. Rev.Saúde Pública, 24:156-63, 1990.

43. VERAS, R.P. \& COUTINHO, E. Estudo de prevalência de depressão e síndrome cerebral orgânica na população de idosos em três distritos da cidade do Rio de Janeiro. Rev.Saúde Pública, 25:209-17, 1991.

44. VERAS, R.P. \& MURPHY, E. The ageing of the third world tackling the problems of communtiy surveys - Part II: A community survey of the elderly population in Rio de Janeiro: a methodological approach. Int.J.Geriatr.Psychiatry, 6:629-37, 1991.

45. VERAS, R.P. \& MURPHY, E. The ageing of the third world: tackling the problems of community surveys - Part I: Brazil - a young country grows old. Int.J.Geriatr.Psychiatry, 6:619-27, 1991.

46. VERAS, R.P. Brazil is getting older: demographic changes and epidemiological challenges. Rev.Saúde Pública, 25:47688, 1991.

47. VERAS, R. A survey of the health of elderly people in Rio de Janeiro, Brazil. London, 1982. [PhD thesis - Division of Psychiatry, United Medical \& Dental Schools, Guy’s Hospital, University of London].

48. VERAS, R.P. \& DUTRA, S. Envelhecimento da populaçāo brasileira: reflexões e aspectos a considerar quando da definição de desenhos de pesquisa para estudos populacionais. Physis: Rev.Saúde Coletiva, 3:107-26, 1993.

49. WEISSMAN, M.M.; MYERS, J.K.; TISCHLER, G.L. PSychiatry disorders (DSM -III) and cognitive impairment among the elderly in a US urban community. Acta Psychiatr.Scand., 71:366-79, 1985.

50. WORLD HEALTH ORGANIZATION. Scientific Group on the Uses of Epidemiology in Aging, Geneva, 1983. Report. Geneva, 1984. (WHO-Technical Report Series 706).

51. WORLD HEALTH ORGANIZATION. Scientific Group on Dementia in Late Life: Research and Action, Paris, 1993. Report. Geneva, 1986. (WHO - Technical Report Series 730).

52. ZHANG, M.; KATZMAN, R.; SALMON, D. The prevalence of dementia and Alzheimer's disease in Shanghai, China: impact of age, gender and education. Ann.Neurol. , 27:428$37,1990$. 


\begin{tabular}{|c|c|c|c|}
\hline \multicolumn{4}{|c|}{ ANEXO } \\
\hline \multicolumn{4}{|c|}{$\begin{array}{l}\text { A Tabela deste Anexo é composta de itens individuais da escala de SCO. Vê-se a } \\
\text { percentagem para cada item nos três distritos. A escala SCO compõe-se, } \\
\text { principalmente, de questões concernentes à memória, orientação e novo aprendizado. } \\
\text { Na Tabela pode-se ver nitidamente que os idosos de Santa Cruz têm desempenho pior, } \\
\text { cometendo mais erros nesses itens. }\end{array}$} \\
\hline \multicolumn{4}{|c|}{$\begin{array}{l}\text { Tabela. Taxas percentuais positivos por distrito - SCO. Espectro: } 0 \text { (sem debilidade } \\
\text { cognitiva) - } 9 \text { (debilidade cognitiva grave). }\end{array}$} \\
\hline Itens & $\begin{array}{l}\text { Copacabana } \\
N=252\end{array}$ & $\begin{array}{l}\text { Méier } \\
N=244\end{array}$ & $\begin{array}{l}S . \text { Cruz } \\
N=242\end{array}$ \\
\hline Idade obviamente errada por "testemunha confiável" & 0,0 & 0,4 & 0,8 \\
\hline Data de nascimento obviamente errada & 4,4 & 8,6 & 16,9 \\
\hline Endereço errado & 3,6 & 4,1 & 12,4 \\
\hline Não sabe há quanto tempo mora naquele endereşo & 4,0 & 3,3 & 9,5 \\
\hline Nāo lembra o nome do presidente do Brasil & 7,1 & 9,4 & 24,8 \\
\hline Não sabe em que mês estamos & 4,4 & 4,5 & 12,4 \\
\hline Não sabe 0 ano em que estamos & 4,0 & 11,5 & 29,3 \\
\hline Teste mão-orelha & 3,2 & 8,2 & 14,5 \\
\hline Não lembra o nome do instituto da pesquisa & 31,0 & 55,3 & 66,9 \\
\hline
\end{tabular}

